\title{
Los efectos de largo plazo de la asimetría y persistencia en la predicción de la volatilidad: evidencia para mercados accionarios de América Latina
}

\author{
The long-run effects of the asymmetry and persistency in forecasting \\ process of the volatility: Regional evidence for Latin America \\ stock markets
}

\author{
Raúl de Jesús Gutiérrez ${ }^{\mathrm{a}, *}$, Edgar Ortiz Calisto ${ }^{\mathrm{b}}$ \\ y Oswaldo García Salgado ${ }^{\text {a }}$ \\ ${ }^{a}$ Universidad Autónoma del Estado de México, México \\ b Universidad Nacional Autónoma de México, México
}

Recibido el 3 de julio de 2015; aceptado el 4 de diciembre de 2015

Disponible en Internet el 23 de junio de 2016

\begin{abstract}
Resumen
Este trabajo propone una extensión al modelo CGARCH a fin de recoger las características de asimetría y persistencia de largo plazo, e investiga sus efectos en el modelado y predicción de la volatilidad condicional de los mercados accionarios de la región de América Latina en el periodo del 2 de enero de 1992 al 31 de diciembre de 2014. En el análisis dentro de la muestra, los resultados estimados de la familia de modelos CGARCH indican la presencia de efectos asimétricos significativos y persistencia de corto y largo plazos en la estructura de la volatilidad de los rendimientos accionarios. Los resultados empíricos también muestran que el uso de medidas simétricas y asimétricas y la prueba estadística de Hansen (2005) son excelentes alternativas para evaluar el poder predictivo de los modelos CGARCH asimétricos. La incorporación de la asimetría y persistencia de largo plazo en la ecuación de la varianza mejora significativamente las predicciones de la volatilidad fuera de la muestra para los mercados accionarios emergentes de Argentina y México.
\end{abstract}

\footnotetext{
* Autor para correspondencia.

Correo electrónico: rjg2005mx@yahoo.com.mx (R. de Jesús Gutiérrez).

La revisión por pares es responsabilidad de la Universidad Nacional Autónoma de México.
} 
Derechos Reservados ( 2016 Universidad Nacional Autónoma de México, Facultad de Contaduría y Administración. Este es un artículo de acceso abierto distribuido bajo los términos de la Licencia Creative Commons CC BY-NC-ND 4.0.

Códigos JEL: C22; C32; C51; C52

Palabras clave: Volatilidad asimétrica; Mercados accionarios emergentes; Medidas de errores simétricas y asimétricas; Prueba de poder predictivo superior

\begin{abstract}
This article proposes an extension to the CGARCH model in order to capture the characteristics of short-run and long-run asymmetry and persistence, and examine their effects in modeling and forecasting the conditional volatility of the stock markets from the region of Latin America during the period from 2 January 1992 to 31 December 2014. In the sample analysis, the estimation results of the CGARCH-class model family reveal the presence of short-run and long-run significant asymmetric effects and long-run persistency in the structure of stock price return volatility. The empirical results also show that the use of symmetric and asymmetric loss functions and the statistical test of Hansen (2005) are sound alternatives for evaluating the predictive ability of the asymmetric CGARCH models. In addition, the inclusion of longrun asymmetry and long-run persistency in the variance equation improves significantly the out of sample volatility forecasts for emerging stock markets of Argentina and Mexico.

All Rights Reserved (C) 2016 Universidad Nacional Autónoma de México, Facultad de Contaduría y Administración. This is an open access item distributed under the Creative Commons CC License BY-NC-ND 4.0.
\end{abstract}

JEL classification: C22; C32; C51; C52

Keywords: Asymmetric volatility; Emerging stock markets; Symmetric and asymmetric loss functions; Superior predictive ability test

\title{
Introducción
}

El nuevo milenio ha sido testigo de la transformación y rápido crecimiento de los mercados accionarios en las economías emergentes. En el contexto de globalización e integración financiera, los mercados accionarios de América Latina han experimentado asombrosas tasas de crecimiento por encima de las economías desarrolladas, en gran parte gracias a las reformas implementadas por autoridades de la región a finales de los años ochenta y principios de los noventa que contribuyeron a la liberalización de los mercados de capital, lo que favoreció la captación de importantes flujos de inversión extranjera hacia estos mercados emergentes y significó cambios fundamentales en sus estructuras financieras (Bekaert y Harvey, 2003). Así mismo, la mayor presencia de inversionistas institucionales para administrar los sistemas de ahorro para el retiro constituye por mucho otro de los factores imprescindibles que explican la reciente evolución de los mercados accionarios de la región de América Latina.

Sin embargo, la creciente incertidumbre internacional generada por la crisis financiera global de Estados Unidos y la crisis de deuda soberana de Europa ha interrumpido el dinámico desempeño financiero en los mercados accionarios emergentes con fuertes ajustes a la baja de las cotizaciones bursátiles. La naturaleza global de las recientes crisis financieras se ha caracterizado por la escasez de liquidez, incremento del riesgo y alta volatilidad, lo que ha afectado negativamente los rendimientos de los participantes en mercados accionarios emergentes con economías frágiles y diferentes características estructurales en sus sistemas financieros en comparación a la estructura más liquida y eficiente de los países desarrollados (Yang, Hsiao, Li y Wang, 2006). 
En periodos de turbulencia financiera el comportamiento de la volatilidad suele ser más persistente antes de alcanzar su nivel más bajo. Este fenómeno típico de los mercados accionarios emergentes generalmente se atribuye a importantes factores macroeconómicos, como fluctuaciones erráticas de los tipos de cambio, crisis financieras y desequilibrios en los sistemas económicos y políticos (Caner y Onder, 2005; Abrugi, 2008; Ikoku, Chukwunonso y Okany, 2014). Así, en periodos largos de inestabilidad la presencia de nueva información ha sido considerada por expertos y académicos como la principal fuente de volatilidad y vulnerabilidad en los mercados financieros internacionales en las últimas décadas (Engle, Ghysels y Sohn, 2013; Vitor, 2015). Consiguientemente, la tarea de entender el comportamiento natural de la volatilidad y su intensidad en los mercados accionarios emergentes se ha convertido en un desafío y prioridad entre los académicos, instituciones financieras, inversionistas individuales e institucionales, porque cuando la volatilidad se define como el principal indicador de la incertidumbre, al mismo tiempo se transforma en un componente clave en el proceso de la toma de decisiones.

El modelado y predicción apropiada de la volatilidad es un factor importante en la selección y administración de portafolios convencionales, por el simple hecho de que los inversionistas institucionales e intermediarios financieros la utilizan como parámetro en la determinación del nivel de riesgo que están dispuestos a aceptar durante el horizonte de tiempo de la inversión, en tanto que en la administración de riesgos y determinación óptima de reservas de capital coadyuva a estimar y solventar las pérdidas de la posiciones de mercado de las instituciones financieras ante cambios inesperados en los factores de riesgo. También, la correcta estimación de la estructura de la volatilidad es fundamental en la implementación de modelos de valuación para las primas de las opciones financieras, porque los operadores requieren del conocimiento de ella para monitorear la dinámica del activo subyacente desde que se inicia el contrato hasta su vencimiento. Finalmente, el pronóstico razonable de la volatilidad puede servir como termómetro del grado de vulnerabilidad y fragilidad de los sistemas financieros y, por ende, de la eficiente asignación de capital en mercados accionarios altamente volátiles.

Desde la publicación del trabajo seminal de Engle (1982), el modelo autorregresivo de heterocedasticidad condicional, y su extensión generalizada por Bollerslev (1986) en el modelo GARCH, han sido reconocidos en la literatura financiera moderna para explicar las características de la volatilidad en las series financieras de frecuencia alta, comúnmente conocidas como volatilidad en aglomeraciones ${ }^{1}$, persistencia y el incuestionable exceso de curtosis. Aunque existen bastantes estudios que favorecen el desempeño de los modelos GARCH para la predicción de la volatilidad (Brailsford y Faff, 1996; Andersen y Bollerslev, 1998; McMillan, Speight y Apgwilym, 2000; McMillan y Speigth, 2004), los resultados empíricos aún no son convincentes en cuanto al poder predictivo fuera de la muestra. Para los mercados accionarios de Tokio y Singapur, Tse (1991) y Tse y Tung (1992) proporcionan sólida evidencia empírica en contra del desempeño de los modelos GARCH en la predicción de la volatilidad fuera de la muestra. Asimismo, Figlewski (1997) sustenta que los modelos de promedios móviles reportan mejores predicciones de la volatilidad fuera de la muestra. Estudios recientes basados en el estadístico de bi-correlación (Hinch-Portmanteau) muestran la ineficiencia de los modelos GARCH para mostrar la estructura estadística de los mercados accionarios de América Latina y Asia (Lim, Melvin, Hinich y Liew, 2005; Bonilla y Sepúlveda, 2011).

\footnotetext{
${ }^{1}$ La volatilidad en aglomeraciones es incuestionablemente una de las características más importantes en los mercados financieros, porque su formación es generada principalmente por la participación de operadores racionales con diferentes horizontes de inversión y su interacción estratégica.
} 
Otra de las debilidades del modelo GARCH, que afecta la estimación y predicción de la volatilidad condicional, se refiere a la asimetría de los choques positivos y negativos de la misma magnitud o efecto de apalancamiento. Black (1976) fue de los primeros en discutir el problema de la asimetría en la volatilidad, al demostrar que las malas noticias intensifican el nivel de la volatilidad y las buenas noticias la disminuyen. Desde entonces, varios modelos de volatilidad asimétrica se han desarrollado para recoger el impacto asimétrico de la información reciente en el mercado; entre ellos se mencionan el modelo GARCH exponencial (EGARCH) de Nelson (1991) y el modelo GARCH-GJR desarrollado por Glosten, Jaganathan y Runkle (1993).

El estudio de la volatilidad asimétrica en los mercados accionarios de economías emergentes es aún limitado en la literatura en comparación con los países industrializados, particularmente en la región de América Latina. López (2004) evalúa el desempeño predictivo de una familia de modelos ARCH, y encontró evidencia de que el modelo EGARCH proporciona el mejor ajuste para explicar la dinámica de la volatilidad futura de los rendimientos del Índice de Precios y Cotizaciones de la Bolsa Mexicana de Valores bajo diferentes medidas de errores predictivos, aunque sus resultados no tienen un sustento estadístico robusto. Alberg, Shalit y Yosef (2008) asumen diferentes distribuciones en las innovaciones de los rendimientos, y demuestran que los modelos GARCH asimétricos presentan el mejor desempeño de predicción para recoger las características de la volatilidad asimétrica y persistencia en el mercado accionario de Israel. Para los mercados accionarios de Sudán y Turquía, Ahmed y Suliman (2011) y Gökbulut y Pekkaya (2014) confirman un elevado grado de persistencia en el proceso de la varianza y la presencia de efectos de apalancamiento en los rendimientos accionarios. En fases de relativa calma del mercado accionario de Malasia, Lim y Sek (2013) confirmaron el poder predictivo del modelo GARCH; sin embargo, en periodos de crisis se ve superado por las especificaciones asimétricas.

En su mayoría, la evidencia empírica señala que los modelos GARCH asimétricos se encuentran abiertos, en teoría, a la interpretación natural del comportamiento de la magnitud de los efectos asimétricos y persistencia de choques en la volatilidad temporal. No obstante, investigaciones recientes han documentado ampliamente que los mercados accionarios emergentes están más expuestos a experimentar eventos extraordinarios como devaluaciones cambiarias (Chue y Cook, 2008, Walid, Chaker, Masoud y Fry, 2011); crisis financieras (Llaudes, Salman y Chivakul, 2010); desplomes bursátiles y especulación (Khwaja y Mian, 2005; Brugger, 2010), y cambios políticos y sociales (Chen, Bin y Chen, 2005). En este respecto, el efecto contagio de crisis bursátiles de mercados maduros hacia mercados accionarios emergentes es un factor clave que detona mayor volatilidad y efectos negativos en estos mercados (Tasdemir y Yalama, 2014). La duración de este tipo de eventos no solo genera información negativa de pánico entre los inversionistas para apresurarlos a liquidar sus portafolios, sino también cambios bruscos en la estructura de la volatilidad en el corto y largo plazos, lo que reduce la capacidad de los modelos GARCH, EGARCH y GARCH-GJR para recoger la asimetría y el grado persistencia en el largo.

Desde un marco de heterocedasticidad condicional autorregresiva, Engle y Lee (1999) propusieron el modelo GARCH de 2 componentes $(\mathrm{CGARCH})$ para estudiar el grado de persistencia en la estructura de la volatilidad; su principal bondad es la capacidad para descomponer la volatilidad condicional en 2 componentes: temporal (corto plazo) y permanente (largo plazo), ante la presencia de operadores racionales heterogéneos o información heterogénea. El componente permanente, modelado como el proceso de largo plazo o tendencia, representa el impacto de las innovaciones generado por cambios esperados en los fundamentos económicos y que presenta el comportamiento de la persistencia en la volatilidad en el largo plazo. En cambio, el componente temporal tiene la función de recoger las fluctuaciones de eventos aleatorios o choques desestabilizadores que experimentan frecuentemente los mercados financieros. Esto es, desviaciones en el 
nivel de equilibrio de la volatilidad en el largo plazo. Aunque Christoffersen, Jacobs, Ornthanalai y Wang (2008) han aportado argumentos concluyentes sobre el poder predictivo de la especificación CGARCH para mostrar la dinámica de la volatilidad. Sin embargo, aún existe la preocupación al momento de pronosticar la volatilidad condicional fuera de la muestra, porque la especificación estándar omite por completo el impacto de la asimetría de los choques informacionales en el componente temporal y permanente de las varianzas.

El principal objetivo de este trabajo es extender el modelo CGARCH para analizar la importancia de la información asimétrica y persistencia de largo plazo, e investigar su efecto en el modelado y predicción de la volatilidad condicional. El estudio aporta importantes contribuciones a la literatura. En primer lugar, el modelo CGARCH se extiende para recoger los efectos de asimetría y persistencia en el corto y largo plazos bajo los procesos EGARCH y TGARCH. Segundo, los modelos CGARCH asimétricos son ajustados para predecir la volatilidad de los rendimientos de los 6 mercados accionarios emergentes más importantes de la región de América Latina en el periodo del 2 de enero de 1992 al 31 de diciembre de 2014. Tercero, el desempeño fuera de la muestra de los modelos GARCH estándar, CGARCH y CGARCH asimétricos se evalúa en el periodo 2010-2014 y bajo 4 medidas de errores predictivos a través de la prueba de poder predictivo superior de Hansen (2005).

Los resultados empíricos dentro de la muestra revelan que en los rendimientos de los mercados accionarios de la región de América Latina se observan efectos de asimetría y persistencia en el corto y largo plazos en la estructura de la volatilidad, particularmente en los países de Chile, Colombia, Perú y México. Estos hallazgos sustentan que choques negativos como crisis financieras y desplomes bursátiles incrementan la volatilidad de corto y largo plazos. Los resultados robustos de la prueba de poder predictivo superior respalda el poder predictivo de los modelos CGARCH asimétricos para predecir la volatilidad fuera de la muestra en los mercados accionarios de Argentina y México.

El resto del trabajo está estructurado de la siguiente forma: la siguiente sección resume la metodología que incluye los modelos CGARCH simétricos y asimétricos, las medidas de errores y la prueba de poder predictivo superior para la evaluación de los modelos de volatilidad. Posteriormente se presenta la descripción de los datos y se resumen los principales hallazgos obtenidos, para acabar con las conclusiones finales.

\section{Modelos GARCH de dos componentes}

En esta sección se presenta otro enfoque alternativo para capturar las características comunes de asimetría y persistencia de corto y largo plazos en la volatilidad condicional.

\section{Modelo GARCH estándar}

El modelo GARCH $(1,1)$ estándar propuesto por Bollerslev (1986) en la literatura sobre volatilidad es una alternativa generalizada del modelo ARCH de Engle (1982). En este contexto, la modelación de la media condicional y varianza condicional está gobernada por:

$$
\begin{aligned}
& r_{t}=\mu+\phi r_{t-1}+\sqrt{h_{t}} z_{t}, \quad z_{t} \sim N(0,1) \\
& h_{t}=\omega+\alpha \varepsilon_{t-1}^{2}+\beta h_{t-1}
\end{aligned}
$$

donde $\mu$ es la media condicional, $h_{t}$ expresa la varianza condicional que depende de la última innovación de los residuales cuadrados $\varepsilon_{t-1}^{2}$, también conocido como efecto ARCH y la varianza 
condicional previa $h_{t-1}, \omega$ es un término determinístico, y su función permite que la varianza condicional alcance un valor positivo en la medida en que el grado de persistencia determinado por $\alpha+\beta$ es menor a 1. El término AR(1) o autorregresivo de orden 1 es agregado a la ecuación de la media condicional, dado que los rendimientos presentes están altamente correlacionados con rendimientos distantes en el tiempo.

Una de las deficiencias del modelo GARCH es que no permite diferenciar los patrones de decaimiento de la persistencia en la volatilidad de corto y largo plazos, por lo que se sugiere un modelo que logre capturar la elevada persistencia en la volatilidad.

\section{Modelo CGARCH de dos componentes}

Engle y Lee (1999) proponen el modelo CGARCH como alternativa para capturar la propiedad de alta persistencia en la volatilidad de los rendimientos financieros. La aproximación permite descomponer la volatilidad condicional en 2 componentes, y señala adecuadamente el comportamiento de decaimiento de la persistencia en la volatilidad de corto y largo plazos.

La especificación del modelo CGARCH $(1,1)^{2}$ se define como:

$$
\begin{aligned}
& r_{t}=\mu+\phi r_{t-1}+\sqrt{h_{t} z_{t}} \\
& h_{t}=q_{t}+\alpha\left(\varepsilon_{t-1}^{2}-q_{t-1}\right)+\beta\left(h_{t-1}-q_{t-1}\right) \\
& q_{t}=\omega+\rho\left(\varepsilon_{t-1}^{2}-h_{t-1}\right)+\delta\left(q_{t-1}-\omega\right)
\end{aligned}
$$

donde $h_{t}$ indica el nivel de la volatilidad de corto plazo que captura las innovaciones, alimentado por eventos exógenos relacionados a aspectos económicos, geopolíticos, incluso de carácter especulativo, y que fluctúa en forma cíclica, $q_{t}$ representa la volatilidad de largo plazo o tendencia, la cual converge al nivel de la volatilidad incondicional $\omega$ a la velocidad de $(\alpha+\beta)<\delta<1$. El término $\left(\varepsilon_{t-1}^{2}-h_{t-1}\right)$ funciona como la potencia dinámica para los movimientos de la tendencia, y la diferencia entre la varianza condicional y su tendencia $\left(h_{t-1}-q_{t-1}\right)$ es el componente temporal que converge a cero a una velocidad $(\alpha+\beta)$.

El modelo CGARCH recoge los efectos de corto y largo plazos de la persistencia, pero su capacidad se reduce ante la presencia de efectos asimétricos, debido al hecho de que choques negativos tienen diferente impacto en la volatilidad que choques positivos de la misma magnitud, no solo en el corto plazo, sino también en el largo plazo.

\section{Modelo CTGARCH asimétrico}

La flexibilidad del modelo CGARCH permite capturar los efectos asimétricos en el corto y largo plazos, con solo agregar parámetros de asimetría a su estructura econométrica, esto es, utilizando los resultados de la especificación TGARCH propuesta por Glosten et al. (1993). La estructura asimétrica o modelo CTGARCH captura los efectos de asimetría de la siguiente manera:

$$
\begin{aligned}
& r_{t}=\mu+\phi r_{t-1}+\sqrt{h_{t} z_{t}} \\
& h_{t}=q_{t}+\alpha\left(\varepsilon_{t-1}^{2}-q_{t-1}\right)+\gamma\left(I\left(\varepsilon_{t-1}<0\right) \varepsilon_{t-1}^{2}-q_{t-1}\right)+\beta\left(h_{t-1}-q_{t-1}\right)
\end{aligned}
$$

\footnotetext{
${ }^{2}$ Para una explicación técnica más detallada del modelo CGARCH, véase Maheu (2005).
} 


$$
q_{t}=\omega+\rho\left(\varepsilon_{t-1}^{2}-h_{t-1}\right)+\psi\left(I\left(\varepsilon_{t-1}<0\right) \varepsilon_{t-1}^{2}-h_{t-1}\right)+\delta\left(q_{t-1}-\omega\right)
$$

donde la variable dummy está gobernada por la función indicadora de Heaviside $I(\bullet)$, la cual es igual a 1 si $\varepsilon_{t-1}<0$ y cero en cualquier otro caso. El efecto de asimetría es observado si $\gamma>0$ y $\psi>0$, lo que indica mayor impacto de las malas noticias con valores $(\alpha+\gamma)$ y $(\rho+\psi)$ sobre $\varepsilon_{t-1}^{2}$ en el corto y largo plazos y el efecto de las noticias optimistas es medido por $\alpha$ y $\rho$.

\section{Modelos CEGARCH asimétrico}

Otra extensión del modelo CGARCH para capturar los efectos asimétricos en el corto y largo plazos es propuesta en este estudio bajo la estructura EGARCH de Nelson (1991).

El modelo CEGARCH tiene la siguiente forma:

$$
\begin{aligned}
& r_{t}=\mu+\phi r_{t-1}+\sqrt{h_{t} z_{t}} \\
& h_{t}=q_{t}+\alpha\left(\varepsilon_{t-1}-q_{t-1}\right)+\gamma\left(\left|\varepsilon_{t-1}\right|-q_{t-1}\right)+\beta\left(h_{t-1}-q_{t-1}\right) \\
& q_{t}=\omega+\rho\left(\varepsilon_{t-1}-h_{t-1}\right)+\psi\left(\left|\varepsilon_{t-1}\right|-h_{t-1}\right)+\beta_{1}\left(q_{t-1}-\omega\right)
\end{aligned}
$$

donde los parámetros de asimetría $\gamma$ y $\psi$ son negativos, a diferencia del modelo CTGARCH, lo que indica mayor impacto de las noticias malas en las volatilidades de corto y largo plazos que las noticias buenas de la misma magnitud. El efecto total en las volatilidades de corto y largo plazos es de $(\alpha-\gamma)\left|\varepsilon_{t-1}\right|$ y $(\rho-\psi)\left|\varepsilon_{t-1}\right|$ si $\varepsilon_{t-1}<0$ y $(\alpha+\gamma)\left|\varepsilon_{t-1}\right|$ y $(\rho+\psi)\left|\varepsilon_{t-1}\right|$ cuando $\varepsilon_{t-1}>0$.

\section{Evaluación del desempeño predictivo de los modelos de volatilidad}

En esta sección se presentan las medidas de errores para la evaluación del desempeño predictivo de los modelos de volatilidad. En general, este proceso se lleva a cabo fuera de la muestra porque los participantes en los mercados accionarios se interesan más en la capacidad de reacción de los modelos cuando llega nueva información al mercado.

Las medidas de errores predictivos se clasifican en simétricas y asimétricas. Entre las medidas simétricas más comunes se encuentran el error cuadrático medio (MSE) y el error absoluto medio (MAE), las cuales son definidas de la siguiente forma:

$$
\begin{aligned}
& \text { MSE }=T^{-1} \sum_{t=1}^{T}\left(h_{t}-\hat{h}_{t}\right)^{2} \\
& \text { MAE }=T^{-1} \sum_{t=1}^{T}\left|h_{t}-\hat{h}_{t}\right|
\end{aligned}
$$

donde $T$ indica el número de predicciones, $h_{t}$ es una variable proxy para la volatilidad no observable, que generalmente se obtiene de los rendimientos cuadrados, y $\hat{h}_{t}$ es la volatilidad estimada a través de las diferentes especificaciones GARCH.

En un estudio analítico, Patton (2011) demostró que estas medidas son robustas para minimizar el error predictivo. Sin embargo, ninguna de ellas proporciona información adicional sobre la asimetría en los errores; esto es, cuando los modelos subestiman o sobreestiman la volatilidad no observable, por lo que la relajación de la asimetría en los errores puede tener consecuencias 
trascendentales para los inversionistas institucionales con posiciones financieras largas y cortas, así como para los compradores y vendedores de opciones sobre índices bursátiles.

De acuerdo con Brailsford y Faff (1996), las medidas de errores asimétricas asignan diferente peso a las predicciones subestimadas y sobrestimadas de la volatilidad de magnitud similar, y son definidas de la siguiente forma:

$$
\begin{aligned}
& \operatorname{MME}(U)=T^{-1}\left[\sum_{t=1}^{O}\left|h_{t}-\hat{h}_{t}\right|+\sum_{t=1}^{U} \sqrt{\left|h_{t}-\hat{h}_{t}\right|}\right] \\
& \operatorname{MME}(O)=T^{-1}\left[\sum_{t=1}^{O} \sqrt{\left|h_{t}-\hat{h}_{t}\right|}+\sum_{t=1}^{U}\left|h_{t}-\hat{h}_{t}\right|\right]
\end{aligned}
$$

donde $U$ y $O$ representan las subestimaciones y sobrestimaciones, y su suma indica el número total de predicciones, $T$.

Investigaciones recientes sobre predicción de la volatilidad fuera de la muestra han demostrado empíricamente el poder de las medidas de errores en la generación de información sobre la evaluación de los modelos estimados. No obstante, uno de los inconvenientes de las medidas es que, a diferencia de las pruebas de contraste de hipótesis, no permiten un análisis robusto en un marco estadístico. Esto, debido a que no se puede concluir que el desempeño predictivo entre 2 modelos estimados es significativamente diferente desde un punto de vista estadístico, con el simple hecho de comparar sus errores predictivos. Para relajar este problema, el presente trabajo emplea la prueba de poder predictivo superior (PPS) de Hansen (2005). Esta prueba estadística robusta permite evaluar el desempeño de 2 o más modelos estimados, a diferencia de las pruebas estadísticas de Diebold y Mariano (1995) y White (2000).

En este contexto, la evaluación predictiva de los modelos se lleva a cabo con base en las medidas de errores, puesto que el modelo base (mejor aproximación) es seleccionado por la medida con error predictivo más pequeño. La prueba estadística PPS consiste en determinar el modelo con el mejor desempeño predictivo. En el periodo $t$, el desempeño predictivo superior del modelo alternativo $k$ en relación al modelo base se define como:

$$
d_{k, t}=L_{0, t}-L_{k, t}, \quad k=1, \ldots, m ; \quad t=1, \ldots, n
$$

donde $L_{0, t}$ es el error de predicción, como se determinó en las ecuaciones (12), (13), (14) y (15) para el modelo base $M_{0}$, y $L_{k, t}$ es el error de predicción asociado a cada modelo alternativo $M_{k}$.

Bajo el supuesto de que el vector $d_{k, t}$ es estrictamente estacionario, la hipótesis nula de interés, de que ninguno de los modelos alternativos alcanza superior desempeño predictivo en relación con el modelo base, puede plantearse como:

$$
H_{0}: \mu_{\max } \equiv \max _{k=1, \ldots, m} \mu_{k} \leq 0
$$

Aquí, el uso del estimador $\mu_{k} \equiv E\left[d_{k, t}\right]$ permite reducir el impacto de los modelos con desempeño predictivo débil, pero al mismo tiempo controla el impacto de los modelos alternativos con $\mu_{k}=0$, como documentó Hansen (2005).

$$
\mu_{k}^{c}=\bar{d}_{k} 1_{\left\{\sqrt{n} \bar{d}_{k} / \hat{\omega}_{k} \geq-\sqrt{2 \log \log n}\right\}}, \quad k=1, \ldots, m
$$


donde $1_{\{\bullet\}}$ es una función indicadora. Además, un resultado inmediato del supuesto de estacionalidad es que la selección del umbral $\sqrt{2 \log \log n}$ garantiza la consistencia del estimador $\mu_{k}^{c}$ para $n$ lo suficientemente grande, incluso para los modelos alternativos con $\mu_{k}=0$.

En consecuencia, el estadístico de la hipótesis nula está definido por:

$$
T_{n}^{P P S}=\max \left\{\left(\max _{k=1, \ldots, m} \frac{\sqrt{n} \bar{d}_{k}}{\hat{\omega}_{k}}\right), 0\right\}
$$

donde $\hat{\omega}_{k}^{2}$ es un estimador consistente de $\omega_{k}^{2} \equiv \lim _{n \rightarrow \infty} \operatorname{Var}\left(\sqrt{n} \bar{d}_{k}\right)$ y $\bar{d}_{k}=n^{-1} \sum_{t=1}^{n} d_{k, t}$.

Para la estimación de $\omega_{k}^{2} \equiv \lim _{n \rightarrow \infty} \operatorname{Var}\left(\sqrt{n} \bar{d}_{k}\right)$ y la probabilidad del estadístico $T_{n}^{P P S}$, Hansen (2005) sugiere el uso del procedimiento bootstrap estacionario basado en Politis y Romano (1994) para obtener la distribución empírica del estadístico de contraste bajo la hipótesis nula, definido por la siguiente expresión:

$$
Z_{k, b, t}^{*}=d_{k, b, t}^{*}-g\left(\bar{d}_{k}\right)
$$

donde $b=1, \ldots, B$ determina el número de muestras bootstrap del vector $d_{k, t}$ para $k=1, \ldots, m$

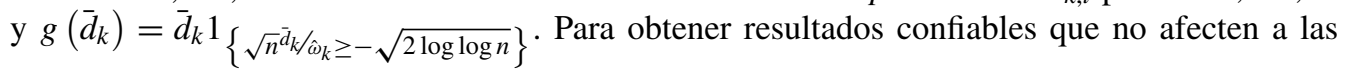
muestras actuales, $B$ debe ser muy grande.

El valor de la probabilidad de la prueba PPS se calcula en 2 etapas. En primer lugar, se obtienen los valores del estadístico $T_{b, n}^{P P S *}$ para cada una de las muestras bootstrap $b=1, \ldots, B$, la cual se define como

$$
T_{b, n}^{P P S^{*}}=\max \left\{\left(\max _{k=1, \ldots, m} \frac{\sqrt{n} \bar{Z}_{k, b}^{*}}{\hat{\omega}_{k}}\right), 0\right\}
$$

donde $\bar{Z}_{k, b}^{*}=n^{-1} \sum_{t=1}^{n} Z_{k, b, t}^{*}, k=1, \ldots, m$.

Por último, los valores de los estadísticos $T_{n}^{P P S}$ y $T_{b, n}^{P P S *}$ son comparados para obtener el valor de la probabilidad bootstrap, es decir:

$$
\operatorname{valor}_{p}^{P P S}=\sum_{b=1}^{B} \frac{1_{\left\{T_{b, n}^{P S *}>T_{n}^{P P S}\right\}}}{B}
$$

La hipótesis nula se rechaza cuando las probabilidades alcanzan valores pequeños.

\section{Aplicación a los mercados accionarios de América Latina}

\section{Descripción y análisis preliminar de los datos}

Si bien los mercados accionarios de los países emergentes se caracterizan por experimentar alta volatilidad, efectos de asimetría y un elevado grado de persistencia, su estudio se ha concentrado en el comportamiento temporal de las características comunes de la volatilidad, por lo que hace falta un estudio sobre los efectos de asimetría y persistencia de largo plazo en la volatilidad. El presente trabajo investiga el efecto de la asimetría y persistencia de largo plazo en la predicción 


\begin{tabular}{lcccccc}
\hline & Argentina & Brasil & Chile & Colombia & Perú & México \\
\hline Media & 0.0231 & 0.2498 & 0.0409 & 0.0720 & 0.1063 & 0.0668 \\
Desviación estándar & 2.2565 & 2.6681 & 0.7768 & 1.2791 & 1.5371 & 1.6180 \\
Máximo & 16.1243 & 28.8332 & 9.0583 & 14.6232 & 12.8231 & 12.1523 \\
Mínimo & -14.7623 & -17.2143 & -5.0174 & -11.0564 & -11.4421 & -14.3112 \\
Sesgo & -0.2432 & 0.3766 & 0.1533 & 0.0617 & 0.1073 & 0.0323 \\
Curtosis & 7.6134 & 10.4123 & 11.4123 & 14.9321 & 11.2956 & 8.6181 \\
Jarque-Bera & 4178 & 10784 & 13762 & 27633 & 13340 & 6129 \\
& $(0.0000)$ & $(0.0000)$ & $(0.0000)$ & $(0.0000)$ & $(0.0000)$ & $(0.0000)$ \\
Q(10) & 76.4344 & 67.4502 & 457.0249 & 376.5091 & 390.4215 & 89.3936 \\
& $(0.0000)$ & $(0.0000)$ & $(0.0000)$ & $(0.0000)$ & $(0.0000)$ & $(0.0000)$ \\
Q ${ }^{2}(10)$ & 1397.8912 & 1166.3421 & 2170.8942 & 1960.0792 & 2797.0961 & 894.2035 \\
& $(0.0000)$ & $(0.0000)$ & $(0.0000)$ & $(0.0000)$ & $(0.0000)$ & $(0.0000)$ \\
Efectos ARCH & 565.958 .2 & 511.142 .4 & 885.434 .8 & 996.2745 & 1049.6192 & 480.9626 \\
& $(0.0000)$ & $(0.0000)$ & $(0.0000)$ & $(0.0000)$ & $(0.0000)$ & $(0.0000)$ \\
\hline
\end{tabular}

Figura 1. Estadísticas básicas de los rendimientos accionarios.

Nota: Las estadísticas básicas de los rendimientos de los mercados accionarios de América Latina son expresadas en porcentajes para el periodo del 2 de enero de 1992 al 31 de diciembre de 2012. Los valores entre paréntesis indican las probabilidades de las pruebas estadísticas Jarque-Bera, efectos ARCH y Ljung-Box Q(10), Q ${ }^{2}(10)$ para los rendimientos simples y cuadrados.

Fuente: Elaboración propia con información de la base de datos de Bloomberg.

de la volatilidad condicional utilizando los precios diarios de los 6 mercados accionarios más importantes de la región de América Latina: Argentina, Brasil, Chile, Colombia, Perú y México ${ }^{3}$. El análisis cubre el periodo del 2 de enero de 1992 al 31 de diciembre de 2014, con una muestra de aproximadamente 5,951 rendimientos diarios. Las series de los precios de los índices accionarios fueron obtenidos de la base de datos de Bloomberg.

La figura 1 presenta las estadísticas básicas de los rendimientos accionarios. Al parecer, todos los índices accionarios presentan propiedades muy similares con rendimientos promedios positivos, lo que se justifica por la tendencia común al alza de los precios bursátiles durante el periodo de análisis, como se muestra en la figura 2. No obstante, la desviación estándar de los rendimientos es relativamente alta, lo que implica una mayor exposición al riesgo para los participantes en estos mercados accionarios, particularmente en Argentina y Brasil.

Todas las series financieras, con excepción de Argentina, muestran las características típicas de asimetría positiva y exceso de curtosis, lo que indica que los choques positivos son más frecuentes que los negativos y distribuciones de rendimientos leptocúrticas, con colas más anchas y largas que la distribución normal, en particular la cola superior. El fenómeno de la no-normalidad de la distribución es también confirmado por el valor del estadístico Jarque-Bera. Con respecto a los estadísticos de Ljung-Box Q(10) para correlación serial, los resultados muestran que la hipótesis de que no existe autocorrelación de orden 10 es rechazada por los pequeños valores de la probabilidad, lo que confirma la presencia de dependencia lineal en los rendimientos accionarios. Asimismo, la correlación serial significativa estadísticamente en los rendimientos cuadrados, determinada por $\mathrm{Q}^{2}(10)$, implica que existe dependencia no lineal en las series de los rendimientos de todos los mercados accionarios.

\footnotetext{
${ }^{3}$ Estos índices bursátiles han sido incluidos en estudios relacionados con los mercados accionarios de América Latina. Últimamente no se incluye al mercado venezolano debido a discontinuidades y falta de confiabilidad en su información.
} 

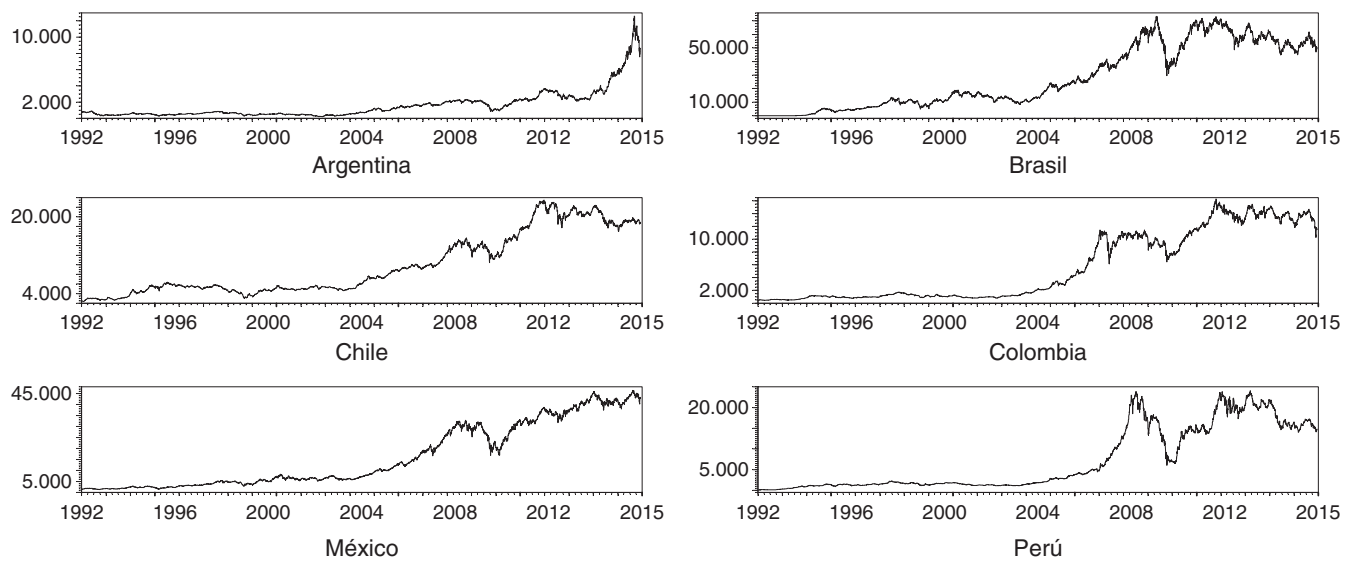

Figura 2. Comportamiento dinámico de los índices accionarios de América Latina. Fuente: elaboración propia con información de la base de datos de Bloomberg.

Además, existe la presencia fuerte de heterocedasticidad condicional o efectos $\mathrm{ARCH}$ en todas las series de los rendimientos financieros, la cual es apoyada por la significancia del estadístico de la prueba del multiplicador de Lagrange a un nivel del 5\%. Esta característica común en los rendimientos financieros es ampliamente sustentada por la figura 3, en donde se puede apreciar fuerte evidencia de volatilidad en aglomeraciones. También se puede observar que la intensidad de la volatilidad es más persistente cuando los precios de los índices accionarios tienden a descender que cuando se incrementan.

En conclusión, el análisis preliminar de los datos sugiere el uso de procesos GARCH de 2 componentes que logren capturar los efectos de asimetría y el fenómeno de persistencia de corto y largo plazos en las innovaciones de los rendimientos accionarios.
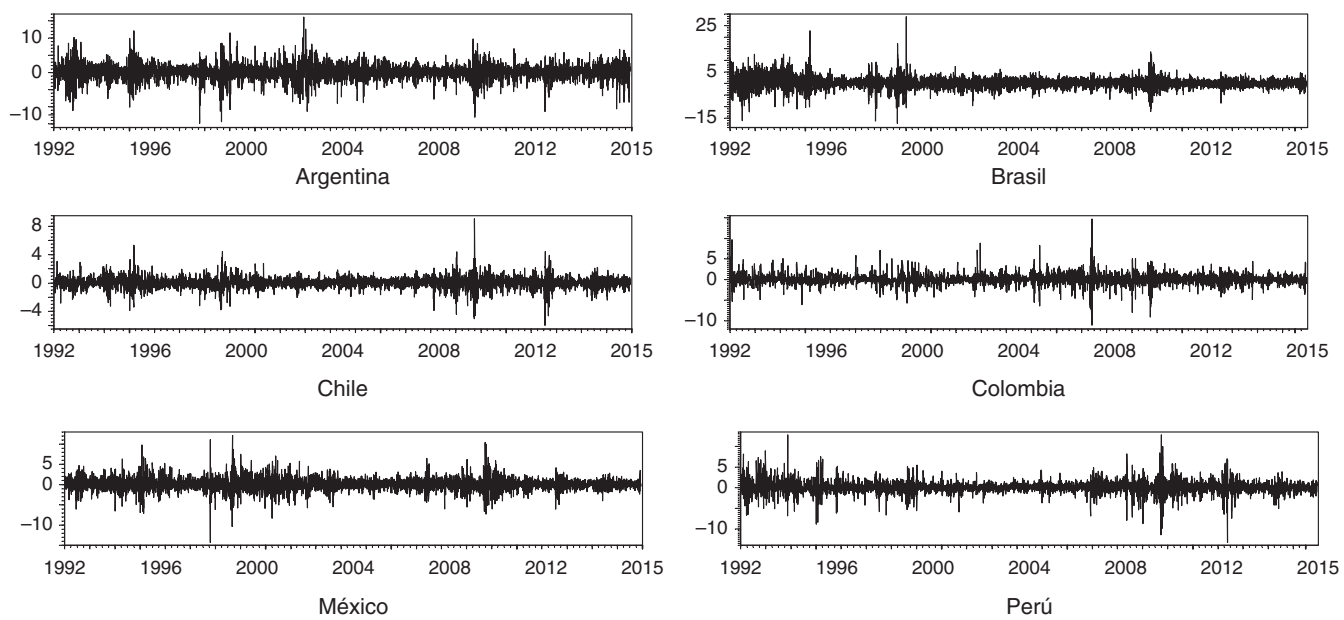

Figura 3. Rendimientos de los índices accionarios de América Latina.

Fuente: elaboración propia con información de la base de datos de Bloomberg. 


\begin{tabular}{|c|c|c|c|c|c|c|c|c|c|c|c|}
\hline & $\mu$ & $\phi$ & $\omega$ & $\alpha$ & $\beta$ & $\rho$ & $\delta$ & $\gamma$ & $\psi$ & $\mathrm{Q}(36)$ & $\mathrm{Q}^{2}(36)$ \\
\hline \multicolumn{12}{|l|}{ México } \\
\hline \multirow[t]{2}{*}{$\mathrm{GARCH}$} & $0.1129 *$ & $0.1404 *$ & $0.0649^{*}$ & $0.1215^{*}$ & $0.8572^{*}$ & & & & & 43.8605 & 33.8912 \\
\hline & $(0.0191)$ & $(0.0155)$ & $(0.0072)$ & $(0.0058)$ & $(0.0066)$ & & & & & [0.1726] & [0.5693] \\
\hline \multirow[t]{2}{*}{ CGARCH } & $0.1117^{*}$ & $0.1401^{*}$ & $0.0006 *$ & $0.1287^{*}$ & $0.7960 *$ & $0.0041^{*}$ & $0.9863^{*}$ & & & 44.1422 & 26.9675 \\
\hline & $(0.0189)$ & $(0.0157)$ & $(0.0002)$ & $(0.0073)$ & $(0.0147)$ & $(0.0012)$ & $(0.0030)$ & & & [0.1653] & [0.8619] \\
\hline \multirow[t]{2}{*}{ CEGARCH } & $0.0561^{*}$ & $0.1332^{*}$ & $-0.0083^{*}$ & $0.0743^{*}$ & 0.9939* & $0.1166^{*}$ & $0.8522^{*}$ & $-0.4985^{*}$ & $-0.9579 *$ & 40.5226 & 29.4436 \\
\hline & $(0.0181)$ & $(0.0147)$ & $(0.0021)$ & $(0.0101)$ & $(0.0015)$ & $(0.0172)$ & $(0.0269)$ & (0.0922) & $(0.1737)$ & {$[0.2776]$} & [0.7720] \\
\hline \multirow[t]{2}{*}{ CTGARCH } & $0.0632^{*}$ & $0.1376^{*}$ & $0.0008^{*}$ & 0.0053 & $0.8082^{*}$ & $0.0061^{* * *}$ & $0.9834^{*}$ & $0.2086^{*}$ & -0.0016 & 41.9601 & 26.3728 \\
\hline & $(0.0184)$ & $(0.0150)$ & $(0.0002)$ & $(0.0091)$ & $(0.0151)$ & $(0.0026)$ & $(0.0034)$ & $(0.0143)$ & $(0.0041)$ & [0.2282] & [0.8799] \\
\hline \multicolumn{12}{|l|}{ Brasil } \\
\hline \multirow[t]{2}{*}{ GARCH } & $0.1845^{*}$ & $0.0411^{*}$ & $0.0804^{*}$ & $0.1083^{*}$ & $0.8815^{*}$ & & & & & 32.9234 & 22.6354 \\
\hline & $(0.0268)$ & $(0.0149)$ & $(0.0105)$ & $(0.0060)$ & $(0.0063)$ & & & & & [0.0010] & [0.0318] \\
\hline \multirow[t]{2}{*}{ CGARCH } & $0.1863^{*}$ & $0.0479^{*}$ & $0.0035^{*}$ & $0.1139 *$ & $0.8148^{*}$ & $0.0084^{* * *}$ & $0.9706^{*}$ & & & 30.8342 & 16.2364 \\
\hline & $(0.0274)$ & $(0.0153)$ & $(0.0013)$ & $(0.0084)$ & $(0.0208)$ & $(0.0038)$ & $(0.0076)$ & & & [0.0029] & [0.1914] \\
\hline \multirow[t]{2}{*}{ CEGARCH } & $0.2031^{*}$ & $0.0664^{*}$ & -0.0157 & $0.1478^{*}$ & $0.9810^{*}$ & 0.0155 & $0.8146^{*}$ & -0.0023 & -0.9999 & 30.9816 & 70.5612 \\
\hline & $(0.0299)$ & $(0.0142)$ & $(0.0244)$ & $(0.0193)$ & $(0.0030)$ & $(0.0235)$ & $(0.2777)$ & $(0.0488)$ & (2.0828) & [0.0019] & [0.0000] \\
\hline \multirow[t]{2}{*}{ CTGARCH } & $0.1198^{*}$ & $0.0565^{*}$ & $0.0063^{*}$ & $-0.0327^{* *}$ & $0.7857^{*}$ & $0.0415^{*}$ & $0.9555^{*}$ & $0.2632^{*}$ & -0.0462 & 30.6012 & 13.1623 \\
\hline & $(0.0279)$ & $(0.0151)$ & $(0.0012)$ & $(0.0158)$ & $(0.0211)$ & $(0.0092)$ & $(0.0063)$ & $(0.0227)$ & $(0.0125)$ & [0.0023] & [0.1945] \\
\hline \multicolumn{12}{|l|}{ Argentina } \\
\hline \multirow[t]{2}{*}{$\mathrm{GARCH}$} & $0.0917^{*}$ & $0.0791^{*}$ & $0.1221^{*}$ & $0.1188^{*}$ & $0.8582^{*}$ & & & & & 13.3881 & 5.8677 \\
\hline & $(0.0252)$ & $(0.0160)$ & $(0.0091)$ & $(0.0055)$ & $(0.0054)$ & & & & & [0.1407] & [0.7448] \\
\hline \multirow[t]{2}{*}{ CGARCH } & $0.0870^{*}$ & $0.0843^{*}$ & $0.0062^{*}$ & $0.1223^{*}$ & $0.7800^{*}$ & $0.0102-$ & $0.9638^{*}$ & & & 11.7367 & 3.8713 \\
\hline & $(0.0261)$ & $(0.0165)$ & $(0.0021)$ & $(0.0081)$ & $(0.0246)$ & $(0.0041)$ & $(0.0089)$ & & & [0.2216] & [0.9155] \\
\hline \multirow[t]{2}{*}{ CEGARCH } & 0.0318 & $0.0843^{*}$ & $-0.0094^{* * *}$ & $0.1271^{*}$ & $0.9808^{*}$ & 0.0362 & $0.8695^{*}$ & -0.0450 & -1.0000 & 10.8982 & 4.8459 \\
\hline & $(0.0265)$ & (0.0149) & $(0.0057)$ & $(0.0224)$ & $(0.0036)$ & $(0.0265)$ & $(0.0697)$ & $(0.0743)$ & $(0.8606)$ & [0.2748] & [0.7856] \\
\hline \multirow[t]{2}{*}{ CTGARCH } & 0.03561 & $0.0933^{*}$ & $0.0056^{*}$ & -0.0147 & $0.7863^{*}$ & $0.0298^{*}$ & $0.9643^{*}$ & $0.2446 *$ & -0.0356 & 10.8982 & 5.3543 \\
\hline & $(0.0264)$ & $(0.0158)$ & $(0.0012)$ & $(0.0117)$ & $(0.0171)$ & $(0.0061)$ & $(0.0058)$ & $(0.0200)$ & $(0.0086)$ & [0.2075] & [0.5674] \\
\hline
\end{tabular}

Figura 4. Estimaciones de los modelos de volatilidad de Argentina, Brasil y México.

Nota: $\mathrm{Q}(36)$ y $\mathrm{Q}^{2}(36)$ indican los valores de los estadísticos de la prueba de Ljung-Box para los residuales simples y cuadrados con 36 rezagos y sus probabilidades son reportadas entre corchetes cuadrados. Los términos *, ** y *** indican significancia al 1, al 5 y al 10\%, respectivamente. Los errores estándar se reportan entre paréntesis.

Fuente: estimaciones propias con información de la base de datos de Bloomberg.

\section{Resultados estimados de los diferentes modelos de volatilidad}

En este trabajo, el modelo CGARCH de Engle y Lee (1999) se extiende a fin de investigar si las características de asimetría y persistencia de largo plazo ejercen efectos en la predicción de la volatilidad condicional de los rendimientos de los mercados accionarios de la región de América Latina. Los parámetros de los modelos de volatilidad son estimados dentro de la muestra utilizando el periodo de estudio del 2 de enero de 1992 al 31 de diciembre de 2009, y asumiendo que los residuales son independientes e idénticamente distribuidos bajo una distribución normal.

Los resultados de los parámetros estimados bajo las 4 estructuras GARCH y sus pruebas de diagnóstico sobre los residuales estandarizados simples y cuadrados son reportados en la figura 4 para los índices de Argentina, Brasil y México y en la figura 5 para los índices de Chile, Colombia y Perú. Los estimadores de $\mu$ que corresponden a la especificación de la media condicional son significativos estadísticamente a un nivel del 1\%, excepto para los modelos CGARCH asimétricos de los mercados accionarios de Argentina y Colombia. Todos los parámetros $\phi$ del proceso 


\begin{tabular}{|c|c|c|c|c|c|c|c|c|c|c|c|}
\hline & $\mu$ & $\phi$ & $\omega$ & $\alpha$ & $\beta$ & $\rho$ & $\delta$ & $\gamma$ & $\psi$ & $Q(36)$ & $Q^{2}(36)$ \\
\hline \multicolumn{12}{|l|}{ Chile } \\
\hline \multirow[t]{2}{*}{$\mathrm{GARCH}$} & $0.0356^{*}$ & $0.3151^{*}$ & $0.0155^{*}$ & $0.1503^{*}$ & $0.8229^{*}$ & & & & & 104.7641 & 34.9479 \\
\hline & $(0.0082)$ & $(0.0133)$ & $(0.0019)$ & $(0.0088)$ & 0.0093 & & & & & [0.0000] & [0.5185] \\
\hline \multirow[t]{2}{*}{ CGARCH } & $0.0349 *$ & $0.3171^{*}$ & $0.0001^{* *}$ & $0.1665^{*}$ & $0.7601^{*}$ & $0.0027^{*}$ & $0.9874^{*}$ & & & 103.8048 & 31.5627 \\
\hline & $(0.0082)$ & $(0.0137)$ & $(0.0000)$ & $(0.0117)$ & $(0.0180)$ & $(0.0009)$ & $(0.0034)$ & & & {$[0.0000]$} & [0.6796] \\
\hline \multirow[t]{2}{*}{ CEGARCH } & $0.0242^{*}$ & $0.3192^{*}$ & $-0.0193^{*}$ & $0.1147^{*}$ & $0.9912^{*}$ & $0.2296^{*}$ & $0.8162^{*}$ & -0.0598 & $-0.1971^{*}$ & 101.5595 & 36.7613 \\
\hline & $(0.0080)$ & $(0.0139)$ & $(0.0060)$ & $(0.0170)$ & $(0.0023)$ & $(0.0218)$ & $(0.0387)$ & $(0.0500)$ & $(0.0584)$ & {$[0.0000]$} & [0.4334] \\
\hline \multirow[t]{2}{*}{ CTGARCH } & $0.0254^{*}$ & $0.3208^{*}$ & $0.0001^{*}$ & $0.1171^{*}$ & $0.7553^{*}$ & $0.0034^{* *}$ & $0.9867^{*}$ & $0.0939^{*}$ & -0.0009 & 103.9484 & 28.5053 \\
\hline & $(0.0084)$ & $(0.0140)$ & $(0.0000)$ & $(0.0126)$ & $(0.0189)$ & $(0.0016)$ & $(0.0034)$ & $(0.0174)$ & $(0.0027)$ & {$[0.0000]$} & [0.8087] \\
\hline \multicolumn{12}{|l|}{ Perú } \\
\hline \multirow[t]{2}{*}{$\mathrm{GARCH}$} & $0.0646^{*}$ & $0.2571^{*}$ & $0.0720^{*}$ & $0.2428^{*}$ & $0.7437^{*}$ & & & & & 106.7912 & 38.8992 \\
\hline & $(0.0147)$ & $(0.0146)$ & $(0.0053)$ & $(0.0101)$ & $(0.0081)$ & & & & & {$[0.0000]$} & {$[0.3405]$} \\
\hline \multirow[t]{2}{*}{$\mathrm{CGARCH}$} & $0.0715^{*}$ & $0.2571^{*}$ & $0.0012^{*}$ & $0.2534^{*}$ & $0.6560^{*}$ & $0.0033^{*}$ & $0.9818^{*}$ & & & 110.7595 & 31.9062 \\
\hline & $(0.0146)$ & $(0.0146)$ & $(0.0003)$ & $(0.0121)$ & $(0.0160)$ & $(0.0009)$ & $(0.0038)$ & & & {$[0.0000]$} & [0.6637] \\
\hline \multirow[t]{2}{*}{ CEGARCH } & $0.0433^{*}$ & $0.2711^{*}$ & $-0.0172^{*}$ & $0.1059^{*}$ & $0.9912^{*}$ & $0.3468^{*}$ & $0.8046^{*}$ & $-0.0808^{* * *}$ & $-0.1434^{*}$ & 104.8657 & 28.5118 \\
\hline & $(0.0145)$ & $(0.0144)$ & $(0.0038)$ & $(0.0150)$ & $(0.0019)$ & (0.0202) & $(0.0240)$ & $(0.0444)$ & (0.0353) & {$[0.0000]$} & [0.8085] \\
\hline \multirow[t]{2}{*}{ CTGARCH } & $0.0469^{*}$ & $0.2608^{*}$ & $0.0007^{*}$ & $0.1789^{*}$ & $0.6732^{*}$ & $0.0034^{*}$ & $0.9881^{*}$ & $0.1256^{*}$ & -0.0031 & 110.6171 & 31.895 \\
\hline & $(0.0151)$ & $(0.0147)$ & $(0.0002)$ & $(0.0143)$ & $(0.0147)$ & $(0.0009)$ & $(0.0025)$ & $(0.0190)$ & $(0.0014)$ & {$[0.0000]$} & [0.6642] \\
\hline \multicolumn{12}{|l|}{ Colombia } \\
\hline \multirow[t]{2}{*}{ GARCH } & $0.0427^{*}$ & $0.3082^{*}$ & $0.1705^{*}$ & $0.2610^{*}$ & $0.6343^{*}$ & & & & & 76.9716 & 197.6593 \\
\hline & $(0.0138)$ & $(0.0152)$ & $(0.0094)$ & $(0.0135)$ & $(0.0155)$ & & & & & {$[0.0001]$} & {$[0.0000]$} \\
\hline \multirow[t]{2}{*}{$\mathrm{CGARCH}$} & $0.0353^{*}$ & $0.2972^{*}$ & $0.0054^{*}$ & $0.3155^{*}$ & $0.4958^{*}$ & $0.0105^{*}$ & $0.9577^{*}$ & & & 93.1709 & 158.0654 \\
\hline & $(0.0127)$ & $(0.0150)$ & $(0.0010)$ & $(0.0180)$ & $(0.0247)$ & $(0.0018)$ & $(0.0053)$ & & & [0.0000] & {$[0.0000]$} \\
\hline \multirow[t]{2}{*}{ CEGARCH } & $0.0294^{* *}$ & $0.3078^{*}$ & $-0.0244^{*}$ & $0.0982^{*}$ & $0.9765^{*}$ & $0.3530^{*}$ & $0.7263^{*}$ & 0.0303 & $-0.1437^{*}$ & 84.5628 & 148.5464 \\
\hline & $(0.0128)$ & $(0.0146)$ & $(0.0045)$ & $(0.0140)$ & $(0.0041)$ & $(0.0234)$ & $(0.0260)$ & $(0.0389)$ & $(0.0347)$ & {$[0.0000]$} & {$[0.0000]$} \\
\hline \multirow[t]{2}{*}{ CTGARCH } & 0.0249 & $0.3020^{*}$ & $0.0058^{*}$ & $0.2138^{*}$ & $0.5310^{*}$ & $0.0141^{*}$ & $0.9563^{*}$ & $0.1333^{*}$ & -0.0073 & 87.7546 & 171.4701 \\
\hline & $(0.0144)$ & $(0.0154)$ & $(0.0012)$ & $(0.0168)$ & $(0.0250)$ & $(0.0028)$ & $(0.0064)$ & $(0.0253)$ & 0.0026 & {$[0.0000]$} & {$[0.0000]$} \\
\hline
\end{tabular}

Figura 5. Estimaciones de los modelos de volatilidad de Chile, Colombia y Perú.

Nota: Q(36) y $\mathrm{Q}^{2}(36)$ indican los valores de los estadísticos de la prueba de Ljung-Box para los residuales simples y cuadrados con 36 rezagos y sus probabilidades son reportadas entre corchetes cuadrados. Los términos *, ** y *** indican significancia al 1, al 5 y al 10\%, respectivamente. Los errores estándar se reportan entre paréntesis.

Fuente: estimaciones propias con información de la base de datos de Bloomberg.

autorregresivo de orden 1 son positivos y significativos a un nivel del $1 \%$. Este hecho implica que la tendencia en los cambios de los precios accionarios se mantiene en la misma dirección en el siguiente periodo.

En cuanto a los parámetros del proceso de la varianza condicional, todos los modelos capturan exitosamente los patrones dinámicos de la volatilidad condicional de corto plazo: sus parámetros estimados son positivos y significativos estadísticamente en los niveles convencionales, con la excepción del modelo CTGARCH de los índices bursátiles de Argentina y México. La suma de los parámetros $\alpha$ y $\beta$ menor a la unidad indica una considerable persistencia en la volatilidad del componente temporal, especialmente en el modelo GARCH tradicional. De hecho, se puede observar que los coeficientes de persistencia $\alpha+\beta$ alcanzan valores de $0.9655,0.9770$, 0.9772, 0.9787 y 0.9865 para Brasil, Argentina, Chile, México y Perú, respectivamente, aunque los resultados de los modelos de 2 componentes basados en las especificaciones CGARCH y CTGARCH confirman lo contrario. Las estimaciones entre 0.9555 y 0.9881 del parámetro $\delta$, para 
los 6 mercados accionarios de la región de América Latina, revelan claramente que el componente de la volatilidad de largo plazo es más persistente y decae a un ritmo más lento que el componente de la volatilidad de corto plazo. Este hecho se atribuye a que los valores del coeficiente de persistencia son menores a los valores de $\delta$, por ejemplo, 0.9266 contra 0.9874 de Chile y 0.8521 contra 0.9881 de Perú para los modelos CGARCH y CTGARCH, respectivamente. En contraste, los resultados del modelo CEGARCH, en todos los índices bursátiles, denotan que el componente temporal de la volatilidad es más persistente.

Considerando la significancia estadística y el signo de los parámetros de asimetría que capturan el impacto de las noticias en el corto y largo plazos asociados a las crisis financieras, desplomes bursátiles y/o auges económicos, los resultados bajo el modelo $\mathrm{CEGARCH}$ son mixtos, puesto que se observan efectos de asimetría en la volatilidad de corto y largo plazos para los mercados accionarios de México y Perú, y solo el componente de la volatilidad de largo plazo en los índices accionarios de Chile y Colombia. En el caso del modelo CTGARCH, el parámetro positivo y significativo a un nivel del $1 \%$ indica que existe solo asimetría en la respuesta de la volatilidad temporal ante la presencia de crisis financieras y desplomes bursátiles para todos los países de la región de América Latina. En consecuencia, la aplicación de modelos CGARCH asimétricos es claramente justificada por los resultados empíricos.

Por último, el diagnóstico de los residuales estandarizados simples y cuadrados se reporta al final de las figuras 4 y 5. Los resultados de las pruebas de Ljung-Box señalan que la hipótesis nula de ausencia de autocorrelación de orden 36 en los residuales estandarizados es imposible rechazarla a un nivel de significancia del 5\%, lo que implica suficiente evidencia a favor de la especificación correcta de la media condicional para explicar el comportamiento de los rendimientos de los índices accionarios de Argentina y México. En el caso de los residuales estandarizados cuadrados, la insignificancia de los estadísticos de Ljung-Box confirma el buen desempeño de los modelos de volatilidad para corregir la autocorrelación de orden 36 en la ecuación de la varianza condicional de los rendimientos financieros de los países de Argentina y México. Estos hechos indican que no existe evidencia estadísticamente significativa de error de especificación en los modelos GARCH, CGARCH simétrico y asimétricos para indicar la heterocedasticidad exhibida en estos mercados accionarios.

\section{Evaluación fuera de la muestra basada en la prueba de poder predictivo superior}

En esta sección, la evaluación fuera de la muestra de la precisión y eficiencia de los modelos GARCH, CGARCH, CEGARCH y CTGARCH se lleva a cabo en el periodo del 4 de enero de 2010 al 31 de diciembre de 2014. Los parámetros de la ecuación de la varianza condicional son reestimados utilizando una ventana móvil de aproximadamente 4,957 observaciones, esto es, del 2 de enero de 1992 al 31 de diciembre de 2009, lo que implica que se remueve la observación más remota y se agrega la observación más reciente a la muestra. La predicción obtenida se compara con la varianza no observable o proxy para calcular el error de predicción. El proceso es repetido hasta obtener la predicción de la varianza condicional del 31 de diciembre de 2014 para cada mercado accionario. De esta manera, el tamaño de la muestra se mantiene fijo durante la reestimación de los modelos de volatilidad y las predicciones fuera de la muestra no se traslapan.

La figura 6 muestra los resultados de las medidas de los errores de predicción MSE, MAE, MME(U), MMM(O) y las probabilidades de la prueba estadística PPS, las cuales se estimaron sobre una base de 10,000 muestras bootstrap estacionarias de la prueba empírica bajo las diferentes medidas de errores predictivos. En este caso, las probabilidades más altas alcanzadas por cualquier modelo base señalan que la hipótesis nula de que el desempeño predictivo fuera de la muestra 


\begin{tabular}{lcccccccc}
\hline \multicolumn{1}{c}{ MSE } & PPS & MAE & PPS & MME(U) & PPS & MME(O) & PPS \\
\hline Argentina & & & & & & & & \\
GARCH & $67.5338(4)$ & 0.0803 & $3.9361(4)$ & 0.0000 & $3.0240(3)$ & 0.4915 & $2.6062(4)$ & 0.0001 \\
CGARCH & $66.5690(2)$ & 0.5487 & $3.8630(3)$ & 0.8197 & $3.0187(2)$ & 0.3429 & $2.5243(3)$ & 0.7848 \\
CEGARCH & $\mathbf{6 6 . 2 6 2 1 ( 1 )}$ & $\mathbf{0 . 9 1 6 3}$ & $\mathbf{3 . 8 4 3 9 ( 1 )}$ & $\mathbf{0 . 9 9 7 2}$ & $3.0280(4)$ & 0.0727 & $\mathbf{2 . 4 9 9 5 ( 1 )}$ & $\mathbf{0 . 9 9 9 7}$ \\
CTGARCH & $67.3860(3)$ & 0.1226 & $3.8568(2)$ & 0.3998 & $3.0008(1)$ & 0.5547 & $2.5206(2)$ & 0.8149 \\
Brasil & & & & & & & & \\
GARCH & $13.1800(3)$ & $\mathbf{0 . 8 4 0 2}$ & $2.1276(3)$ & $\mathbf{0 . 9 7 3 7}$ & $1.7677(3)$ & 0.5738 & $1.6759(3)$ & 0.7809 \\
CGARCH & $13.0306(2)$ & 0.4735 & $2.0914(2)$ & 0.8333 & $1.7667(2)$ & 0.5948 & $1.6262(2)$ & $\mathbf{1 . 0 0 0 0}$ \\
CEGARCH & $13.2536(4)$ & 0.0530 & $2.1539(4)$ & 0.0000 & $1.7868(4)$ & 0.0000 & $1.6975(4)$ & 0.0000 \\
CTGARCH & $\mathbf{1 2 . 7 0 6 7 ( 1 )}$ & 0.5246 & $\mathbf{2 . 0 7 1 4 ( 1 )}$ & 0.6030 & $\mathbf{1 . 7 4 2 8 ( 1 )}$ & $\mathbf{0 . 7 3 3 0}$ & $\mathbf{1 . 6 2 2 0 ( 1 )}$ & 0.8741 \\
México & & & & & & & & 0.0000 \\
GARCH & $3.6433(4)$ & 0.0053 & $1.0194(4)$ & 0.0000 & $1.0412(4)$ & 0.0000 & $0.8736(4)$ & 0.0000 \\
CGARCH & $3.5906(3)$ & 0.4483 & $0.9699(2)$ & 0.9175 & $1.0154(3)$ & 0.7184 & $0.8178(2)$ & 0.9597 \\
CEGARCH & $\mathbf{3 . 5 2 6 7 ( 1 )}$ & $\mathbf{0 . 4 9 2 0}$ & $\mathbf{0 . 9 6 5 7 ( 1 )}$ & $\mathbf{0 . 9 8 0 7}$ & $1.0138(2)$ & 0.6059 & $\mathbf{0 . 8 1 5 6 ( 1 )}$ & $\mathbf{1 . 0 0 0 0}$ \\
CTGARCH & $3.5706(2)$ & 0.3799 & $0.9723(3)$ & 0.8909 & $\mathbf{1 . 0 0 7 6 ( 1 )}$ & $\mathbf{0 . 9 9 9 9}$ & $\mathbf{0 . 8 2 6 6 ( 3 )}$ & 0.9458 \\
\hline
\end{tabular}

Figura 6. Resultados de la prueba de poder predictivo superior.

Nota: Los valores en negrita indican los modelos de volatilidad con mejor desempeño predictivo fuera de la muestra basado en los valores pequeños de las funciones de pérdidas MSE, MAE, MME(U), MME(O), MME(O) y probabilidades más altas de la prueba PPS.

Fuente: estimaciones propias con información de la base de datos de Bloomberg.

de los modelos alternativos es ampliamente superado por el modelo base, no se puede rechazar. La primera columna de la figura constituye el nombre del modelo base que se comparará con los otros 3 modelos alternativos.

Para el caso de los países de Argentina, Brasil y México, los modelos CEGARCH y CTGARCH permiten predicciones fuera de la muestra de la volatilidad más exactas, a diferencia de los modelos GARCH y CGARCH bajo las 4 medidas MSE, MAE, MME(U) y MME(O). Estos hallazgos son sustentados por los valores pequeños alcanzados en las medidas de errores simétricas y asimétricas. De esta manera, los resultados empíricos sugieren claramente que la volatilidad de los mercados accionarios de Argentina, Brasil y México responde de manera diferente a las noticias buenas y malas, lo que a su vez implica que los choques negativos en estas plazas bursátiles tienen mayor impacto en el corto plazo, pero escasos efectos en el largo plazo.

Por su parte, las probabilidades de la prueba estadística PPS indican significativamente que el modelo CEGARCH presenta mejor desempeño predictivo fuera de la muestra que los modelos alternativos basados en las medidas MSE, MAE y $\mathrm{MME}(\mathrm{O})$ para los mercados accionarios de Argentina y México. En el caso de $\mathrm{MME(U),} \mathrm{el} \mathrm{modelo} \mathrm{CTGARCH} \mathrm{proporciona} \mathrm{el} \mathrm{valor} \mathrm{más}$ alto de la probabilidad de la prueba estadística PPS para todas las predicciones fuera de la muestra considerada. Este hallazgo empírico se atribuye a que la medida asimétrica MMM(U) penaliza las predicciones subestimadas de la volatilidad, que en este caso representan aproximadamente el 73.42 y el $77.28 \%$ de la muestra para Argentina y México, respectivamente. Aunque es importante resaltar que la probabilidad de la prueba estadística PPS del modelo CEGARCH se encuentra por arriba del nivel de significancia del 5\%, lo que significa que aún puede ser considerado un excelente modelo base para predecir la volatilidad futura en el mercado accionario de México, en el caso del mercado accionario de Argentina se tiene como segunda alternativa al modelo CGARCH. Este hallazgo es justificado por la insignificancia del parámetro de asimetría tanto en el corto como en el largo plazo. 
La información generada por las medidas de errores asimétricas es relevante para las estrategias de cobertura con contratos de opciones sobre índices accionarios porque existe una relación positiva entre la volatilidad y la prima de la opción. En este sentido, el alto porcentaje en las predicciones subestimadas de la volatilidad puede proporcionar sesgo en las primas de las opciones, lo que beneficiará directamente a los inversionistas institucionales que mantienen posiciones financieras largas de portafolios réplica sobre índices accionarios, y que en un entorno de incertidumbre tienen la necesidad de protegerlos a través de opciones put, pero al mismo tiempo perjudican a los compradores de opciones.

Por otra parte, si bien los rendimientos de los mercados accionarios de la región de América Latina poseen características similares, la capacidad de los modelos CGARCH simétricos y asimétricos no es suficiente para proporcionar estimaciones precisas de la volatilidad futura en los mercados accionarios de Brasil, Chile, Colombia y Perú. Al analizar los resultados estadísticos de la figura 6 se observa que el modelo CTGARCH alcanza el mejor desempeño predictivo de acuerdo con los valores pequeños de las medidas de errores simétricas y asimétricas. Sin embargo, los resultados de la prueba estadística PPS contradicen dichos hallazgos empíricos porque los modelos GARCH y CGARCH proporcionan las probabilidades más altas bajo los criterios MSE, MAE y $\operatorname{MME}(\mathrm{O})$, respectivamente. Este hecho se atribuye a que ninguno de los modelos de volatilidad logró eliminar la autocorrelación observada en los residuales estandarizados simples y cuadrados en el mercado accionario de Brasil, lo que conlleva a subestimar o sobreestimar la volatilidad futura. ${ }^{4}$

\section{Conclusiones}

En esta investigación, el modelo CGARCH de Engle y Lee (1999) fue extendido a fin de investigar si las características de asimetría y persistencia de largo plazo ejercen efectos en la predicción de la volatilidad condicional de los rendimientos de los mercados accionarios de la región de América Latina. En el análisis empírico dentro de la muestra se utilizaron los logaritmos de los rendimientos accionarios diarios de enero de 1992 a diciembre de 2009, mientras que en el análisis de la evaluación fuera de la muestra se utilizó el periodo de enero de 2010 a diciembre de 2014. La evidencia empírica muestra que en la volatilidad de los rendimientos accionarios se encontraron vestigios de efectos de asimetría de largo plazo en los casos de Chile, Colombia México y Perú; esto significa que los choques negativos no solo tendrán mayor impacto en el componente de la volatilidad de corto plazo, sino también en su tendencia de largo plazo. Así mismo, se encontró que los modelos CGARCH y CTGARCH permiten una modelación flexible del comportamiento asintótico de los rendimientos en los mercados accionarios de la región de América Latina, con implicaciones importantes para la medición de la persistencia de largo plazo en la estructura de la volatilidad. En el marco de la prueba de poder predictivo superior, los resultados estadísticos fuera de la muestra revelan que el modelo CEGARCH proporciona el mejor desempeño para predecir la volatilidad que los modelos alternativos, puesto que es aceptado por 3 de las 4 medidas de errores, esto es, MSE, MAE y MME(O), en tanto que bajo el criterio de la medida asimétrica MME(U), el modelo CTGARCH resulta la mejor opción para la predicción de la volatilidad en los mercados accionarios de Argentina y México. Otro hallazgo empírico importante que debe resaltarse es que todos los modelos estimados subestiman la volatilidad en los

\footnotetext{
${ }^{4}$ Por falta de espacio, los resultados de la prueba estadística PPS no son reportados en el caso de los mercados accionarios de Chile, Colombia y Perú, pero están disponibles para cualquier aclaración. Además, los resultados son inconsistentes, como en el caso del mercado accionario de Brasil.
} 
mercados accionarios considerados. Estos resultados tienen importantes implicaciones financieras para los inversionistas institucionales con posiciones largas y cortas, puesto que los modelos GARCH, CGARCH simétricos y asimétricos suelen ser más apreciados por los vendedores de opciones y menos deseables por los compradores de opciones. Los resultados débiles derivados de los modelos CGARCH simétrico y asimétrico en el caso de los mercados accionarios de Brasil, Chile, Colombia y Perú dejan abierta una agenda de investigaciones futuras sobre la estimación de modelos más complejos, como especificaciones FIGARCH y FIEGARCH que permitan un completo entendimiento de la persistencia de largo plazo en la volatilidad condicional.

\section{Referencias}

Abrugi, A. B. (2008). Empirical relationship between macroeconomic volatility and stock returns: Evidence from Latin American markets. International Review of Financial Analysis, 17(2), 396-410.

Ahmed, A. E. M. y Suliman, S. Z. (2011). Modeling stock market volatility using GARCH models evidence from Sudan. International Journal of Business and Social Science, 2(23), 117-128.

Alberg, D., Shalit, H. y Yosef, R. (2008). Estimating stock market volatility using asymmetric GARCH models. Applied Financial Economics, 18(15), 1201-1208.

Andersen, T. G. y Bollerslev, T. (1998). Answering the skeptics: Yes, standard volatility models do provide accurate forecasts. International Economic Review, 39(4), 889-905.

Bekaert, G. y Harvey, C. R. (2003). Emerging markets finance. Journal Empirical Finance, 10(1-2), 3-55.

Black, F. (1976). Studies of stock market volatility changes. Proceedings of the American Statistical Association, Business and Economic Statistics Section, 177-181.

Bollerslev, T. (1986). Generalised autoregressive conditional heteroskedasticity. Journal of Econometrics, 31(3), 307-327.

Bonilla, C. A. y Sepúlveda, J. (2011). Stock returns in emerging markets and the use of GARCH models. Applied Economics Letters, 18(14), 1321-1325.

Brailsford, T. J. y Faff, R. W. (1996). An evaluation of volatility forecasting techniques. Journal of Banking and Finance, 20(3), 419-438.

Brugger, S. I. (2010). Capital Especulativo y Crisis Bursátil en América Latina, Contagio, Crecimiento y Convergencia (1993-2005) [tesis doctoral]. Facultad de Economía, UNAM.

Caner, S. y Onder, Z. (2005). Sources of stock market volatility in emerging markets. Applied Economics, 37(8), 929-942.

Chen, D. H., Bin, F. S. y Chen, D. S. (2005). The impacts of political events on foreign institutional investors and stock returns: Emerging market evidence from Taiwan. International Journal of Business, 10(10), 165-188.

Christoffersen, P., Jacobs, K., Ornthanalai, C. y Wang, Y. (2008). Option valuation with long-run and short-run volatility components. Journal of Financial Economics, 90(3), 272-297.

Chue, T. K. y Cook, D. (2008). Emerging market exchange rate exposure. Journal of Banking \& Finance, 32(7), $1349-1362$.

Diebold, F. X. y Mariano, R. S. (1995). Comparing predictive accuracy. Journal of Business \& Economic Statistics, 13(3), 253-263.

Engle, R. F. (1982). Autoregresive conditional heteroskedasticity with estimates of the variance of the UK inflation. Econometrica, 50(4), 987-1008.

Engle, R. F. y Lee, G. G. J. (1999). A permanent and transitory component model of stock return volatility. En R. Engle y H. White (Eds.), Cointegration, Causality, and Forecasting: A Festschrift in Honor of Clive W. J. Granger (pp. 475-497). Oxford: Oxford University Press.

Engle, R. F., Ghysels, E. y Sohn, B. (2013). Stock market volatility and macroeconomic fundamentals. The Review of Economics and Statistics, 95(3), 776-797.

Figlewski, S. (1997). Forecasting volatility. Financial Markets Institutions and Instruments, 6(1), 1-88.

Glosten, L., Jaganathan, R. y Runkle, D. (1993). On the relation between the expected value and the volatility of the nominal excess returns on stocks. Journal of Finance, 48(5), 1779-1801.

Gökbulut, R. I. y Pekkaya, M. (2014). Estimating and forecasting volatility of financial markets using asymmetric GARCH models: An application on Turkish financial markets. International Journal of Economics and Finance, 6(4), 23-35.

Hansen, P. R. (2005). A test for superior predictive ability. Journal of Business and Economics Statistics, 23(4), 365-380.

Ikoku, A. L., Chukwunonso, T. y Okany, T. (2014). Did de economic and financial crisis affect stock market sensitivity to macroeconomic risk factors? Evidence from Nigeria and South Africa. Journal of Business, 19(3), 275-290.

Khwaja, A. y Mian, A. (2005). Unchecked intermediaries: Price manipulation in an emerging stock market. Journal of Financial Economics, 78(1), 303- 241. 
Lim, K. P., Melvin, J., Hinich, V. y Liew, K. S. (2005). Statistical inadequacy of GARCH models for Asian stock markets: Evidence and implications. Journal of Emerging Market Finance, 4(3), 263-279.

Lim, C. M. y Sek, S. K. (2013). Comparing the performances of GARCH-type models in capturing the stock market volatility in Malaysia. Procedia Economics and Finance, 5(2), 478-487.

Llaudes, R., Salman, F. y Chivakul, F. (2010). The impact of the great recession on emerging markets. IMF Working Paper wp/10/237.

López, F. (2004). Modelado de la volatilidad y pronóstico del Índice de Precios y Cotizaciones de la Bolsa Mexicana de Valores. Revista de Contaduría y Administración, 49(2), 43-72.

Maheu, J. (2005). Can GARCH models capture long-range dependence? Studies in Nonlinear Dynamics \& Econometrics, 9(4), 1-41.

McMillan, D. G., Speight, A. E. H. y Apgwilym, O. (2000). Forecasting UK stock market volatility. Applied Financial Economics, 10(4), 435-448.

McMillan, D. G. y Speigth, A. E. H. (2004). Daily volatility forecasts: Reassessing the performance of GARCH models. Journal of Forecasting, 23(6), 445-460.

Nelson, D. B. (1991). Conditional heteroscedasticity in asset returns: A new approach. Econometrica, 59(2), 347-370.

Patton, A. J. (2011). Volatility forecast comparison using imperfect volatility proxies. Journal of Econometrics, 160(1), 246-256.

Politis, D. N. y Romano, J. P. (1994). The stationary bootstrap. Journal of the American Statistical Association, 89(428), 1303-1313.

Tasdemir, M. y Yalama, A. (2014). Volatiity spillover effects in interregional equity markets: Evidence from Brazil and Turkey. Emerging Markerts Finance and Trade, 50(2), 190-202.

Tse, Y. K. (1991). Stocks returns volatility in the Tokyo stock exchange. Japan and the World Economy, 3(3), $285-298$.

Tse, Y. K. y Tung, S. H. (1992). Forecasting volatility in the Singapore stock market. Asia Pacific Journal of Management, 9(1), 1-13.

Vitor, G. (2015). Sensitivity, persistence and asymmetric effects in international stock market volatility during the global financial crisis. Revista de Métodos Cuantitativos para la Economía y la Empresa, 19(19), 42-65.

Walid, C., Chaker, A., Masoud, O. y Fry, J. (2011). Stock market volatility and exchange rates in emerging countries: A Markov-state switching approach. Emerging Markets Review, 12(3), 272-292.

White, H. (2000). A reality check for data snooping. Econometrica, 68(5), 1097-1126.

Yang, J., Hsiao, C., Li, Q. y Wang, Z. (2006). The emerging market crisis and stock market linkages: Further evidence. Journal of Applied Econometrics, 21(6), 727-744. 